\title{
La Administración de Justicia en Granada durante el primer tercio del siglo Xx. La acción de los jueces municipales
}

\author{
Salvador Cruz Artacho *
}

\section{INTRODUCCIÓN **}

Durante todo el primer tercio del siglo xx la realidad social granadina está caracterizada por la acción de una tupida red clientelar. Los tentáculos de ésta va a alcanzar la totalidad de las esferas de la vida pública provincial. Una de ellas será la Administración de Justicia, siendo el cuerpo de los Jueces municipales uno de los ámbitos naturales de la actividad caciquil.

Son numerosos los testimonios de denuncia conservados. La gran mayoría de los mismos hacen referencia al ejercicio abusivo que el Juez Municipal hace de su cargo.

Así, en 1919, una protesta contra el Juzgado Municipal de Motril venía a afirmar que «... se cree en Motril, y en la provincia así resulta

* Universidad de Granada.

** Esta comunicación solamente pretende ser una primera aproximación, con carácter de introducción, al ejercicio de la Administración de la Justicia local en la provincia de Granada, considerada ésta como parte integrante y relevante, en casos, de las estrategias de poder de la clase dominante local.

En esta comunicación sólo esbozamos algunas ideas que estudios posteriores analizarán de manera pormenorizada. 
algunas veces, que el Juzgado no es más que un arma política terrible, que hábil o burdamente esgrimida, pero de decisión, reduce a la impotencia al adversario, y cuyo beneplácito es requisito indispensable para que tenga fuerza de obligar y se ejecuten hasta los decretos de los mismos Ministros de la Corona cuanto éstos perjudican a sus contrarios y beneficien las aspiraciones de los que cuentan con la ayuda de Juez ...” ${ }^{1}$.

Esta situación de irregularidad permanente viene reforzada desde el mismo momento en que se lleva a cabo la elección del cargo de Juez Municipal. En los nombramientos de Jueces y Fiscales municipales suelen atenerse única y exclusivamente a las indicaciones de los Gobernadores Civiles de cada provincia. Se forman las "ternas" de candidatos, se emiten los informes respectivos y, posteriormente, el voto consultivo se convierte en acuerdo. La propuesta aprobada es aquella que satisface las exigencias del Gobernador Civil, quien, en numerosos casos, obedece los deseos y dictados del cacique de la zona en cuestión ${ }^{2}$. Este cuerpo de Jueces, pues, no se recluta atendiendo a lo establecido en la normativa legal, sino que "cada uno era designado por el respectivo cacique, conforme a las conveniencias de éste, por ministerio de su otro brazo, el Gobernador Civil, siquiera el nombramiento vaya refrenado, para cubrir la apariencia legal, por el presidente de la Audiencia .... ${ }^{3}$. Esto mismo viene a afirma el señor Bullón de la Torre, quien refiriéndose al cargo de Juez Municipal expresa que "se nombra para administrar justicia a personas de pésimos antecedentes y ninguna ilustración, como recompensa de servicios electorales o como instrumento para prestarlos... que ofrecen garantía de servir el día de la elección para toda clase de atropellos y coacciones ... tienen preferentemente el carácter de agentes electorales .... ${ }^{4}$.

Esto nos lleva a considerar la acción de la administración de Justicia, a nivel municipal y provincial, como uno de los brazos ejecutores al servicio de la estrategia de poder de la clase dominante. La acción legal, como elemento de control social, va a convertirse en un canal de distribución de favores bajo el dictado de los oligarcas y caciques locales que la dirigen.

' Reclamación al Juzgado Municipal de Motril (1919). Cuatrienio 1920-1923. Archivo de la Real Chancillería de Granada. Cabina 701, legajo A-81, Pieza 2-2.

2 Costa Joaquin, Oligarquía y Caciquismo como la forma actual de gobierno en España: urgencia y modo de cambiarla, Volumen I. Zaragoza, Guara editorial, 1982 (reedición del original de 1901).

"Altamira, Rafael y otros, "La Administración de Justicia", en Costa, Joaquín, Op. cit. Volumen II, pág. 95.

${ }^{4}$ Costa, Joaquín, Op. cit. 
Por consiguiente, cuando se pretende analizar «fenómenos» clientelistas, manifestaciones caciquiles, en una determinada comunidad, es necesario atender a la organización y actuación de aparato judicial, puesto que éste constituye uno de los medios con que cuenta la clase dominante para perpetuar el sistema de relaciones sociales existentes en la comunidad. Tengase en cuenta que la red clientelar en la provincia granadina, durante el primer tercio del siglo $\mathrm{xx}$, encuentra dificultades crecientes para su reproducción, recurriendo con suma frecuencia a actos de violencia para perpetuar el status quo social. Es obvio que en estas coyunturas es indispensable controlar los mecanismos de control social, de violencia estatal instrumentalizada; es necesario, en definitiva, controlar la Administración de Justicia en la provincia ${ }^{5}$.

Ya algunos autores regeneracionistas van a señalar a la Administración de Justicia cuando de Caciquismo se hable ${ }^{6}$.

Asi mismo, con asiduidad encontramos en los estudios que se están realizando en la actualidad, referencias y análisis en los que quedan reflejados con suficiente claridad el papel que juegan las autoridades judiciales en los procesos electorales, pues podian «facilitar en gran medida que las elecciones tuvieran una apariencia de legalidad, evitando tener que recurrir a la violencia o a las coacciones más descaradas para lograr el resultado deseado ${ }^{7}$. No menos cierto es, a su vez, que la actividad «irregular» de los Jueces Municipales y Jueces de Instrucción, no se reduce a los períodos en que se desarrollan los procesos electorales; previamente llevan a cabo en la provincia una labor de hostilidades, procesamientos y suspensiónes, en su caso, de aquellas Corporaciones Municipales que desarrollan una acción "contraria» a los dictámenes establecidos por la Oligarquía caciquil de la zona. Posteriormente tendremos ocasión de analizar numerosos casos de lo que aquí expongo ${ }^{8}$.

Por tanto, y a modo de resumen, considero que un análisis de los entresijos del poder, del ejercicio del poder, a nivel local, requiere un

5 Prueba de lo que afirmo es la «lucha» que se puede observar en los procesos electorales; las reclamaciones presentadas ante la Audiencia Provincial y los informes reservados en los que se hace constancia del uso -en el caso en que esto se dé- que dicho candidato hizo del cargo de Juez Municipal en cuatrienios anteriores.

${ }^{6}$ Memoria presentada por Frera, Enrique, en Costa, Joaquín, Op. cit.

7 Barreda Fontes, José María, Caciques y Electores. Ciudad Real, Instituto de Estudios Manchegos, 1986, págs. 348-355.

${ }^{8}$ Véase el apartado de reclamaciones a los candidatos presentados en los procesos electorales. 
estudio pormenorizado de la actividad - en la esfera local- que lleva a cabo la Administración Judicial; creo que no se puede dejar a un lado uno de los «brazos» ejecutores de los designios de la Oligarquia local, detentadora de los aparatos del poder en la comunidad. A su vez, la Administración Judicial no sólo cumple la función de brazo ejecutor, sino que se convierte en el instrumento ideal para mantener el status quo, para perpetuar las relaciones de dominación y las estrategias de poder de la clase dominante.

La Administración de Justicia no cumple la función de elemento modernizador, de difusor de los ideales desarrollados y difundidos desde el corpus ideológico del liberalismo ${ }^{9}$, de elemento dinamizador en los ámbitos rurales; por el contrario, en manos de los oligarcas, ésta se amoldará a las exigencias de los mismos, convirtiendose en elemento que perpetua el fenómeno del Caciquismo en el seno de la realidad social de la provincia.

Una vez reflejado someramente este panorama genérico, es necesario hacer referencia al tipo de fuentes documentales utilizadas y los ámbitos de análisis.

Fundamentalmente, en lo que se refiere a fuentes archivísticas, he utilizado los diversos expedientes de ternas de Jueces Municipales que se encuentran custodiados en el Archivo de la Audiencia Territorial de Granada (Archivo de la Real Chancillería de Granada). Los expedientes conservados comienzan desde 1914 en la gran mayoría de los casos estudiados. También se completa la información que ofrecen dichos expedientes con los listados que nos ofrecen los Boletines Oficiales de la Provincia ${ }^{10}$. En la actualidad, por estar en proceso de catalogación, no he podido acceder a la información que puedan aportar los expedientes personales de dichos Jueces.

Con respecto a los ámbitos, a los términos municipales estudiados, he decidido analizar aquéllos que constituyen Cabeza de Partido Judicial. Una análisis de la totalidad de las circunscripciones judiciales de la provincia, de un lado, haría excesivamente voluminosa la comunicación; de otro, las conclusiones a las que llegaremos no variaran, pues los com-

${ }^{9}$ Me estoy refiriendo a la idea de igualdad de los hombres ante la Justicia.

${ }_{10}$ Los Boletines Oficiales de la Península ofrecen el listado de titulares de los Juzgados Municipales de la Provincia. Este tipo de tistados no ofrece ningún tipo de información complementaria sobre los candidatos que han resultado elegidos. Normalmente, el listado suele aparecer en la segunda quincena del mes de julio. 
portamientos, con las particularidades propias de cada comunidad, son similares en el conjunto del territorio provincial.

Asi pues, los municipios analizados son:

\section{CUADRO I}

Términos Municipales y Partidos Judiciales.

Provincia de Granada.

\begin{tabular}{|c|c|}
\hline Término Municipal & Partido \\
\hline Albuñol & Albuñol \\
\hline Alhama & Alhama \\
\hline Baza ...... & Baza \\
\hline Campillo ..... & Granada \\
\hline Guadix ........ & Guadix \\
\hline Huéscar ....... & Huéscar \\
\hline Iznalloz ..... & Iznalloz \\
\hline Loja............ & Loja \\
\hline Montefrío $\ldots . .$. & Montefrío \\
\hline Motril $\ldots \ldots \ldots \ldots$ & Motril \\
\hline 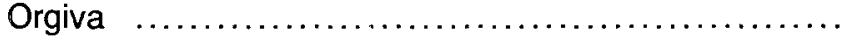 & Orgiva \\
\hline$\ldots \ldots \ldots+\ldots \ldots+\ldots, \ldots$ & Granada \\
\hline Salvador $\ldots . . . \ldots .$. & Granada \\
\hline Santafé & Santafé \\
\hline Ugijar & Ugijar \\
\hline
\end{tabular}

Por último hacer constar que en el análisis no sólo se hace referencia al cargo de Juez Municipal Propietario, sino que se tiene en consideración los diversos nombramientos de Jueces Municipales Suplentes.

\section{CANDIDATURAS Y ELECCIONES A JUEZ MUNICIPAL}

\section{II.1. Candidaturas a Jueces Municipales}

Como he referido anteriormente, en los procesos de elección de los cargos de Juez Municipal Propietario y Suplente no se atiende, por to 
general, a los preceptos legales establecidos. Las disposiciones legales son papel mojado ante la realidad caciquil de la provincia.

De otro lado, hay que añadir que la propia legislación favorece, por el carácter "poco preciso" de la misma, el que se cometan irregularidades en los nombramientos. La ley, en este caso concreto, no sirve a los ideales de «lgualdad y Justicia social», se convierte en un elemento más de control en manos de la oligarquía dirigente.

Téngase presente que los Jueces Municipales y sus Suplentes serán nombrados por las Salas de Gobierno de las respectivas Audiencias, las cuales, para el nombramiento, tendrán muy en consideración las condiciones, los méritos, que ostentan los incluidos en las listas de candidatos para administrar de forma recta la circunscripción. Condiciones éstas que se deducen de los informes que aportan los Presidentes de los Tribunales de Partido sobre las distintas candidaturas.

Estas «imprecisiones" - condiciones de rectitud moral del candidato, etc. - van a permitir un campo propicio a los «manejos turbios de los caciques» en los distintos términos municipales. Va a ser el propio oligarca local, o bien bajo sus designios, el que determine el carácter favorable o desfavorable de los informes que se remiten de los distintos candidatos a Juez.

Irregularidades en la presentación de candidatos y en los nombramientos posteriores se pueden rastrear en la documentación consultada con suma frecuencia.

Así, la legislación, a su vez, establece una serie de condiciones que debe cumplir el candidato elegido. Una de ellas, básica, es la de saber leer y escribir. Pues bien, he encontrado nombramientos de Jueces en los que el titular del Juzgado no cumplía estos mínimos requisitos ${ }^{11}$. Estos casos son, evidentemente, extraordinariamente poco usuales, pero nos constatan las irregularidades que se cometían en la elaboración de los informes de los candidatos.

De otro lado, las consideraciones de carácter político de los candidatos, que harian peligrar el sentido de ecuanimidad en sus acciones, viene a ser considerada como causa determinante de la no elección del candidato. Aun así, encontramios múltiples reclamaciones sobre irregu-

11 Véase el Juzgado Municipal de Acequias. Archivo de la Real Chancillería de Granada, Cabina 701, legajo A-49. 
laridades cometidas en los nombramientos de Jueces que tienen constatada su «militancia» política en la circunscripción.

Esto ya ofrece algunas pautas explicativas, algunos indicios, de la función que cumple dicho cargo de Juez en las estrategias de control del poder por parte de la oligarquía caciquil de la zona.

A su vez, la legislación, como es obvio, da preferencia, a la hora de ocupar el cargo de Juez Municipal, a aquellas personas que pertenecen al Cuerpo de Aspirantes a la Judicatura y a aquellos relacionados con la Carrera Judicial (Abogados, Magistrados, Procuradores, etc.).

Esta última consideración lleva a pensar que la gran mayoría de las candidaturas presentadas contienen a personas pertenecientes al sector profesional está relacionado con la Carrera Judicial.

¿Se respeta esta preferencia en las candidaturas presentadas en los distintos casos anaiizados en la provincia de Granada?

CUADRO II

Relación entre candidaturas a Juez Municipal y profesiones de los aspirantes. (1914-1927)

\begin{tabular}{|c|c|c|}
\hline Profesion & Número & $\begin{array}{c}\text { Porcentaje } \\
(\%)\end{array}$ \\
\hline $\begin{array}{l}\text { Abogados } y \text { demás profesiones relacionadas } \\
\text { con la Carrera Judicial } \ldots \ldots \ldots \ldots \ldots \ldots \ldots \ldots \ldots \ldots\end{array}$ & 244 & 64,9 \\
\hline Aparejadores $\ldots \ldots \ldots \ldots \ldots \ldots \ldots \ldots$ & 1 & 0,2 \\
\hline Carrera Militar & 8 & 2,1 \\
\hline Comerciantes $\ldots \ldots \ldots \ldots \ldots$ & 16 & 4,2 \\
\hline Empleados $\ldots \ldots \ldots \ldots \ldots \ldots \ldots \ldots$ & 4 & 1,0 \\
\hline 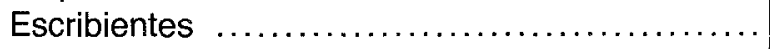 & 1 & 0,2 \\
\hline Estudiantes $\ldots \ldots \ldots \ldots \ldots \ldots \ldots$ & 1 & 0,2 \\
\hline Farmacéuticos & 5 & 1,3 \\
\hline Industriales ....... & 8 & 2,1 \\
\hline Labradores ..... & 4 & 1,0 \\
\hline Médicos & 3 & 0,7 \\
\hline Propietarios & 61 & 16,2 \\
\hline Sacristán & 1 & 0,2 \\
\hline Sin profesión declarada ............ & 19 & 5,0 \\
\hline TOTAL & 376 & 99,3 \\
\hline
\end{tabular}

Fuente: Elaboración propia. 
Como puede deducirse del cuadro II parece que esta preferencia viene respetándose en lo que respecta a la presentación de candidaturas en los diversos procesos de elección acaecidos entre 1914 y 1927 . No en vano abogados y demás profesiones relacionadas con la Carrera Judicial suponen el $64,9 \%$ del total de las candidaturas presentadas (244 sobre 376).

Parece, pues, en este primer análisis, que esta preferencia que establece la legislación a la hora de nombramientos, influye en los sectores profesionales que aportan candidatos. Ésto se puede ver con mayor claridad si se relaciona el cuadro I con el cuadro VI que exponemos más adelante.

No obstante habría que decir que quizás esta tabla nos conduzca a unas conclusiones demasiado genéricas. Sería necesario, a mi entender, establecer otro tipo de tabla que nos puntualizara estos resultados generales.

En este punto de la exposición cabría hacer una consideración: la provincia de Granada, en su conjunto, tiene como denominador común el hecho de poder ser considerada una sociedad eminentemente rural. Los espacios que podríamos denominar urbanos son muy escasos, pudiéndose reducir los mismos, casi exclusivamente, al ámbito de la capital de la provincia ${ }^{12}$.

Por tanto, quizás un análisis comparativo entre ámbito urbano (Granada capital) y ámbito rural (resto de casos considerados), pueda matizarnos estas primeras conclusiones acerca del tipo de candidaturas presentadas. Téngase en cuenta que los hábitos sociales del mundo urbano presentan sustanciales diferencias de las costumbres sociales propias de las comunidades rurales.

Efectivamente, de los datos que nos aporta el cuadro número III podemos deducir notables diferencias entre lo que acontece en el ámbito urbano de la capital de provincia y el resto de los municipios considerados en el estudio.

La diferencia sustancial viene dada, en lo referente a candidaturas, en los porcentajes de «Abogados y demás profesiones relacionadas con la Carrera Judicial». Hecho éste que subraya una vez más la diversidad de comportamientos que se puede encontrar entre el ámbito urbano de la capital y el resto de los lugares estudiados.

12 Consúltense los datos que ofrece CALERO AMOR, Antonio María, en Historia del movimiento obrero en Granada. Madrid, Tecnos, 1973. 
La Administración de Justicia en Granada durante ...

CUADRO II!

Relación de datos entre la Capital y la Provincia

(1914-1927)

\begin{tabular}{|c|c|c|c|c|}
\hline Profesiones & Capital & $\%$ & Provincia & $\%$ \\
\hline $\begin{array}{l}\text { Abogados y demás profesiones re- } \\
\text { lacionadas con la Carrera Judicial }\end{array}$ & 134 & 96,4 & 110 & 46,4 \\
\hline Aparejadores $\ldots \ldots \ldots \ldots \ldots \ldots \ldots$ & 0 & 0,0 & 1 & 0,4 \\
\hline Carrera Militar & 0 & 0,0 & 8 & 3,4 \\
\hline Comerciantes & 0 & 0,0 & 16 & 6,8 \\
\hline Empleados ...... & 0 & 0,0 & 4 & 1,7 \\
\hline Escribientes ....... & 0 & 0,0 & 1 & 0,4 \\
\hline Estudiantes ..... & 0 & 0,0 & 1 & 0,4 \\
\hline Farmacéuticos & 0 & 0,0 & 5 & 2,1 \\
\hline Industriales & 0 & 0,0 & 8 & 3,4 \\
\hline Labradores & 0 & 0,0 & 4 & 1,7 \\
\hline Médicos ... & 0 & 0,0 & 3 & 1,3 \\
\hline Propietarios & 0 & 0,0 & 61 & 25,7 \\
\hline Sacristán ... & 0 & 0,0 & 1 & 0,4 \\
\hline Sin profesión declarada ....... & 5 & 3,6 & 14 & 5,9 \\
\hline N. ${ }^{\circ}$ TOTAL & 139 & 100,0 & 237 & 100,0 \\
\hline
\end{tabular}

Fuente: Elaboración propia

En Granada capital prácticamente la totalidad de las candidaturas provienen del sector profesional de los abogados; por el contrario, en el resto de los términos municipales se produce una mayor diversidad de los estratos socio-profesionales representados en las mismas.

Aun siendo así, también dentro del conjunto de los municipios de la provincia (excluida la Capital) van a existir notables diferencias, en lo que atañe a las candidaturas presentadas. Existen zonas donde los porcentajes de «abogados y ... " son elevadísimos, encontrando, por contra, otras en la que son prácticamente insignificantes y donde se da una gran diversidad de sectores representados.

Motivo a destacar, de otro lado, va a ser el número, elevado, de «Propietarios" que opta a ocupar el sillón de Juez Municipal.

Sin lugar a dudas, los dos estratos profesionales con mayor número de candidaturas son el de Propietarios y el de Profesiones relacionadas con la Carrera Judicial. No todas las zonas estudiadas presentan, evidentemente, las mismas proporciones, muy al contrario, existen notabilísimas diferencias entre las diversas comunidades, entre zonas estudiadas. 
Esto, que en su suma viene a responder a las diversas estrategias que la clase dominante local establece en su lucha por el control de los resortes del poder en la zona, puede verse con suficiente claridad en el cuadro IV, en que ofrecemos los datos de Propietarios y Profesiones relacionadas con la Carrera Judicial.

De esta manera, se pueden encontrar zonas tales como la circunscripción de Loja, donde el sector profesional que proporciona candidatos es el de abogados (100\%); frente a ésta, en el lado opuesto del abanico de posibilidades, zonas como el término municipal de Iznalloz donde el "arco de profesiones» de donde se reclutan los candidatos es muy amplio ${ }^{13}$.

Entre estos dos casos se mueven el resto de los analizados en esta páginas, como puede deducirse del cuadro número IV.

\section{CUADRO IV}

Relación entre Propietarios y Profesiones de la Carrera Judicial en las candidaturas de los términos municipales analizados de la provincia de Granada (Excluida la Capital) (1914-1927)

\begin{tabular}{|c|c|c|c|c|}
\hline Término municipal & Carr. Jud. & $\%$ & Propiet. & $\%$ \\
\hline Albuñol .. & 3 & 20,0 & 5 & 33,3 \\
\hline Alhama & 3 & 33,3 & 4 & 44,4 \\
\hline Baza & 13 & 54,1 & 3 & 12,5 \\
\hline Guadix & 21 & 87,5 & 3 & 12,5 \\
\hline Huéscar .. & 4 & 44,4 & 1 & 11,1 \\
\hline Iznalloz ...... & 1 & 7,6 & 2 & 15,3 \\
\hline Loja ......... & 20 & 100,0 & 0 & 00,0 \\
\hline Montefrío $\ldots \ldots \ldots \ldots \ldots \ldots . . . .$. & 12 & 48,0 & 6 & 24,0 \\
\hline Motril $\ldots . .$. & 16 & 84,2 & 2 & 10,5 \\
\hline Orgiva & 1 & 5,2 & 10 & 52,6 \\
\hline Santafé $\ldots . . . \ldots \ldots$ & 8 & 29,6 & 10 & 37,0 \\
\hline Ugijar $\ldots \ldots \ldots \ldots \ldots \ldots \ldots \ldots \ldots \ldots$ & 10 & 30,3 & 11 & 33,3 \\
\hline
\end{tabular}

Fuente: Elaboración propia.

${ }^{13}$ El término municipal de Iznalloz ofrece los siguientes resultados: Abogados, 1; Propietarios, 2; Comerciantes, 2; Empleados, 2; Estudiantes, 1; Industriales, 1; Labradores, 2; Médicos, 1; Sacristán, 1. 
En resumen, las candidaturas presentadas en los distintos términos municipales no presentan grandes diferencias a lo que viene a ser la norma de comportamiento general en el territorio nacional.

La gran mayoría de los candidatos pertenecen a lo que podríamos definir como «la clase alta»; son propietarios agrícolas, patronos industriales, comerciantes e individuos pertenecientes a las profesiones liberales (Abogados, etc.). Como puede observarse, la gran mayoría de los candidatos estarían incluidos en el grupo de propietarios de los medios de producción.

Las clases medias (empleados, etc.) apenas si están representadas en las candidaturas.

De esto parece deducirse que son los propios sectores de la clase dominante los que optan al control del ejercicio de la Justicia, en aras, como podrá verse más adelante, de perpetuar el status quo social existente en cada zona.

Ahora es necesario analizar los procesos de elección, cuáles son los candidatos elegidos, a qué sectores profesionales están adscritos, qué grado de continuidad se observa en la posesión del cargo de Juez Municipal. Esto nos acercará aún más a un conocimiento más preciso de los entresijos de la Administración de Justicia local en la provincia de Granada.

\subsection{Los procesos de elección de Jueces Municipales}

Con los expedientes conservados en el Archivo de la Chancillería de Granada es posible estudiar perfectamente el espacio cronológico comprendido entre los años 1914-1927. Entre estas dos fechas se pueden encontrar los siguientes cuatrienios, con sus correspondientes procesos de elección al inicio de cada cuatrienio. Ver Cuadro $\mathrm{V}^{14}$. Cuatrienios en los Juzgados Municipales. Provincia de Granada.

${ }^{14}$ En esta tabla solamente se hace referencia a los distintos cuatrienios. El proceso de elección se realiza en el año de comienzo del cuatrienio. A su vez, es necesario advertir que en el curso del cuatrienio, por vacantes, se producen numerosísimos procesos de elección para cubrir las vacantes producidas. 
CUADRO $V$

Cuatrienios en los Juzgados Municipales. Provincia de Granada. (1914-1927)

\begin{tabular}{|c|c|c|c|}
\hline \multicolumn{2}{|c|}{ Grupo I } & \multicolumn{2}{|c|}{ Grupo II } \\
\hline Cuatrienios & Término municipal & Cuatrienios & Término municipal \\
\hline $\begin{array}{l}1916-1919 \\
1920-1923 \\
1924-1927\end{array}$ & $\begin{array}{l}\text { Guadix } \\
\text { Huescar } \\
\text { Iznalloz } \\
\text { Montefrío } \\
\text { Motril } \\
\text { Orgiva } \\
\text { Sagrario } \\
\text { Salvador } \\
\text { Santafé } \\
\text { Ugijar }\end{array}$ & $\begin{array}{l}1914-1917 \\
1918-1921 \\
1922-1925\end{array}$ & $\begin{array}{l}\text { Albuñol } \\
\text { Alhama } \\
\text { Baza } \\
\text { Campillo } \\
\text { Loja }\end{array}$ \\
\hline
\end{tabular}

Como puede observarse existen dos secuencias diferenciadas en la cronología de cuatrienios de los Juzgados Municipales en la provincia.

Esta relación, a su vez, se complica extraordinariamente con las constantes bajas que se producen, en el seno de cada Juzgado municipal en el transcurso del cuatrienio, del cargo de Juez Municipal Propietario o Suplente, lo que conlleva un nuevo proceso de elección del puesto que resulta vacante.

Como he dicho anteriormente, en este corto espacio de tiempo se van a producir un gran número de procesos de elección, caracterizándose los mismos, en primer lugar, por la gran cantidad de candidatos que se presentan para el cargo de Juez Municipal Propietario; en segundo lugar, sobresale la continuidad en los procesos de elección de algunas de las candidaturas que se presentan. Continuidad que no sólo se verá reflejada en los candidatos, sino también en el ejercicio del cargo.

Como exponía en apartados anteriores, se establecía que para ocupar el cargo de Juez Municipal en la capital de circunscripción (los casos estudiados en estas páginas), se preferirian a aquellos candidatos que pertenecieran al Cuerpo de Aspirantes a la Judicatura y al Ministerio Fiscal, Abogados, y demás profesiones relacionadas con la Carrera Judicial. Las candidaturas presentadas parecian alumbrar que esta preferencia se respetaría, al ser mayoritario el grupo de Abogados. 
CUADRO VI ${ }^{15}$

Candidatos elegidos en los cargos de Juez Municipal Propietario y Juez Municipal Suplente. Provincia de Granada. (1914-1927)

\begin{tabular}{|c|c|c|c|c|c|c|}
\hline Profesión & N..$^{\circ}$. & $\%$ & JMP & $\%$ & JMS & $\%$ \\
\hline Abogados y Carrera Judicial & 82 & 65,0 & 46 & 78,0 & 36 & 54,0 \\
\hline Aparejadores ............. & 1 & 0,8 & 0 & 00,0 & 1 & 1,5 \\
\hline Carrera Militar...$\ldots \ldots \ldots$ & 1 & 0,8 & 0 & 00,0 & 1 & 1,5 \\
\hline Comerciantes $\ldots \ldots \ldots \ldots \ldots$ & 6 & 4,8 & 2 & 3,4 & 4 & 6,0 \\
\hline Estudiantes ................ & 1 & 0,8 & 1 & 1,7 & 0 & 00,0 \\
\hline Farmaceúticos $\ldots \ldots \ldots \ldots \ldots \ldots$ & 2 & 1,6 & 0 & 00,0 & 2 & 3,0 \\
\hline Industriales & 5 & 4,0 & 0 & 00,0 & 5 & 7,5 \\
\hline Labradores & 1 & 0,8 & 0 & 00,0 & 1 & 1,5 \\
\hline Médicos... & 2 & 1,6 & 1 & 1,7 & 1 & 1,5 \\
\hline Propietarios $\ldots \ldots \ldots \ldots \ldots \ldots \ldots \ldots$ & 19 & 15,1 & 8 & 13,5 & 11 & 16,4 \\
\hline Total & 126 & 99,3 & 59 & 100,0 & 67 & 98,9 \\
\hline
\end{tabular}

Fuente: Elaboración propia.

¿Cuándo se produce la elección, se respeta esta preferencia?

Sí, como se ha podido ver en los cuadros anteriores, en todos los términos municipales se presentan candidaturas del sector profesional considerado en primer lugar en el cuadro, los resultados que nos alumbra el Cuadro VI indica que esta preferencia se cumple con relativa moderación.

De los 82 «Abogados y demás ...» elegidos, 27 corresponden a los Juzgados de Granada Capital (el $33 \%$ ). En Granada capital, el porcentaje a abogados elegidos es del 96,4 (27 de 28 candidatos). Por el contrario, el porcentaje del $65 \%$ se ve reducido al 55,5\% (55 de 99) para el conjunto de los términos municipales de la provincia (excluida la capital).

Como se observa, las tendencias que se apuntaban cuando se analizaron las candidaturas, se mantienen en las elecciones de los titulares y suplentes de los Juzgados. Tendencias que vienen a subrayar las diferencias existentes entre el ámbito de la Capital y el resto de la provincia.

Diferencias en lo que atañe a los sectores de los que se «extrae» el personal que ocupará los cargos en la Administración de Justicia local,

${ }^{15}$ Las abreviaturas JMP, corresponden al cargo de Juez Municipal Propietario; JMS al cargo de Juez Municipal Suplente. 
pero similitudes en lo que respecta al comportamiento, al ejercicio que de dicha Administración se ejerce. En estas consideraciones ahondaré cuando trate, un poco más adelante, el apartado de reclamaciones y protestas a los procesos de elección.

Otro dato a resaltar es el grado, en algunos casos concretos, de continuidad en el ejercicio del cargo de Juez Municipal. En esta cuestión sí se encuentra grandes diferencias entre distintas zonas. Ejemplo de ello es, de una parte, el caso del Juzgado Municipal de Alhama. En éste, el cargo de Juez Municipal Propietario y Suplente lo ocuparán, durante todo el período analizado, los señores Francisco Gómez y Álvarez Campana y Pedro Pérez Benitez respectivamente. Por el contrario, nos encontramos con casos como el Juzgado Municipal de Montefrío o Iznalloz en los que el cargo nunca será ocupado dos veces por un candidato. Véase el cuadro VII al respecto.

Como era de esperar, el análisis de los resultados de las elecciones viene a confirmar lo que afirmaba en el apartado anterior: la Justicia Municipal estará en manos de la clase dominante.

\section{CUADRO VII}

Elección de Jueces Municipales Propietarios y Suplentes. Grado de continuidad en el cargo.

Provincia de Granada

(1914-1927)

\begin{tabular}{|c|c|c|c|c|}
\hline Término municipal & N. ${ }^{\circ}$ total & Eleg. 1 & Eleg. 2 & Eleg. 3 \\
\hline Albuñol & 9 & 7 & 1 & 0 \\
\hline Alhama $\ldots . . . \ldots$. & 6 & 0 & 0 & 2 \\
\hline Baza & 10 & 4 & 3 & 0 \\
\hline Guadix ... & 8 & 3 & 1 & 1 \\
\hline Granada Capital . . & 28 & 14 & 4 & 2 \\
\hline Huéscar ......... & 5 & 1 & 2 & 0 \\
\hline Iznalloz ... & 6 & 6 & 0 & 0 \\
\hline Loja ....... & 9 & 4 & 1 & 1 \\
\hline Montefrío .. & 10 & 10 & 0 & 0 \\
\hline Motril ..... & 19 & 7 & 3 & 2 \\
\hline Orgiva $. . . \ldots \ldots .$. & 6 & 4 & 1 & 0 \\
\hline Santafé $\ldots . . .$. & 11 & 4 & 1 & $1\left(^{*}\right)$ \\
\hline Ugijar $\ldots \ldots \ldots$ & 11 & 7 & 2 & 0 \\
\hline
\end{tabular}

(*) Este candidato es elegido en 5 ocasiones. 
Resta por analizar los expedientes de reclamaciones e informes reservados que se elevaban a la Audiencia Territorial de Granada con motivo de los procesos de elección. En éstos se hace referencia a la idoneidad o no idoneidad de alguno de los candidatos presentados. Este tipo de documentación ofrece alguna información sobre el uso y abuso en el ejercicio del cargo de Juez Municipal.

\section{3. Reclamaciones e informes reservados}

Los expedientes de elección a Juez Municipal, en numerosas ocasiones, van acompañados de informes reservados y reclamaciones contra la validez de la candidatura ${ }^{16}$.

En este estudio analizaré solamente algunas de las mismas; aquéllas que hacen referencia al ejercicio del cargo de Juez en el municipio como un «arma política».

Ello debido a que es destacable la presencia de un denominador común en la gran mayoría de los casos analizados: «el carácter marcadamente político del candidato".

¿Qué tipo de actitudes concretas son denunciadas en las reclamaciones o en los informes reservados? Podrian resumirse en tres apartados:

1. Relacionadas con su «militancia e intolerancia política».

2. Relacionadas con actividades de obstaculización a las gestiones de las diversas Corporaciones Municipales.

3. Relacionadas con incompatibilidades del candidato por sus actividades profesionales.

El apartado 1 y 2 están en clara relación pues, los procesos ejercidos contra los Ayuntamientos se producen, por norma general, en los momentos previos a comicios electorales.

${ }^{16}$ Generalmente, en todos los procesos electorales que he analizado se encuentran gran cantidad de reclamaciones. La gran mayoría de las mismas son denunciadas de particulares con respecto a la idoneidad del candidato. Los informes reservados conservados son menos numerosos, si bien ofrecen datos cualitativamente importantes sobre la actividad política del candidato en cuestión. 
II. 3.1 «El carácter político de los candidatos»

Este tipo de denuncias son muy constantes y se producen en todas las zonas que se han analizado en este estudio. ¿Qué entendían por "carácter marcadamente político" de los candidatos? ¿qué se denunciaba concretamente?

En términos generales se argumentaba que las circunstancias de ser políticos activos, privaba al cargo de Juez de las condiciones de serena imparcialidad necesarias para el ejercicio del cargo, puesto que el funcionario, con el calor de las luchas políticas, "se apasiona fácilmente y aun sin quererlo, inclina sus resoluciones y falta a su deber, perjudicando derechos e intereses sacratísimos... ${ }^{17}$.

Ejemplo de ésto es la actuación que desarrollará el Juez Municipal Propietario de Loja, D. Ramón Rico Fuensalida, durante el cuatrienio 1918-1921. Este señor tomará parte directa en una contienda electoral escandalosa, interviene cohibiendo a los electores, dando motivo a que se produzcan protestas en el acto del escrutinio general de las elecciones municipales.

Por tanto, los informes reservados y las protestas conservadas en los expedientes llevan a considerar el cargo de Juez como un cargo de carácter marcadamente político en la medida en que el puesto de Juez es utilizado "como arma de combate en vez de paz y de justicia, y (está) sujeto a intereses de bandería, produciendo males irreparables en nuestros derechos..." ${ }^{18}$.

Debo dejar constancia que, en los casos analizados, a pesar de reclamaciones e informes desfavorables, el candidato siempre va a ser elegido para desempeñar el cargo de Juez Municipal. Una vez más, queda patente que es el cacique, el oligarca local, el que antepone sus criterios a aquéllos establecidos en la legislación vigente.

Un caso ejemplificador de lo que afirmo lo constituye el Juzgado Municipal de Motril.

17 Reclamación contra el Sr. D. Ramón Rico Fuensalida. Juez Municipal de Loja (cuatrienio 1918-1921). Archivo de la Real Chancillería de Granada, Cabina número 701, legajo A-76, pieza 3-2.

${ }_{18}$ Reclamación contra el Sr. D. Emilio Guillen Sobrino. Juzgado Municipal de Baza, cuatrienio 1918-1921. Archivo de la Real Chancillería de Granada, Cabina 701, legajo A-55, pieza 4-2. 
Se conservan una cierta cantidad de reclamaciones contra el nombramiento del Sr. Gerardo Trujillo Hernandez y un informe reservado negativo sobre dicho candidato. En el informe aparecen frases tales como "... su defecto único consiste en creer que para los fines políticos se instituyó el Juzgado Municipal de Motril y las interinidades en el Juzgado de instrucción...» o «... el referido Sr. Juez, con descaro inaudito, resuelve los juicios verbales y de faltas a medida de su capricho..." ${ }^{19}$.

Informes y reclamaciones de este talante se pueden encontrar en los expedientes de todos los términos municipales analizados. En éllos, repito una vez más, la figura del Juez Municipal aparece como un cargo político al servicio de intereses partídistas.

Esto me lleva a considerar que, en el estudio de la realidad del poder local, es necesario hacer mención especial al ejercicio de la Justicia, en la medida en que se convierte en arma arrojadiza al servicio de la clase dominante local. Es necesario insertar este ámbito de la Administración Pública dentro de lo que constituye el entramado del poder local. Pretensión ésta que se sale de los márgenes que acotan esta comunicación.

19 Reclamaciones contra el Sr. D. Gerardo Trujillo Hernandez. Juzgado Municipal de Motril. Cuatrienio 1920-1923. Archivo de la Real Chancillería de Granada, Cabina 701, legajo A-81, pieza $2-2$. 